\title{
Performance Analysis of Distributed Spatial Multiplexing with Multi-hop Amplify and Forward Relaying in Cellular Networks
}

\author{
M.Sushanth Babu ${ }^{1}$ and Prof.K.Kishan Rao ${ }^{2}$ \\ ${ }^{1}$ Associate Professor, Department of Electronics \& Communication Engineering \\ Vaagdevi College of Engineering, Warangal, A.P., INDIA \\ sushanth_6@yahoo.com \\ ${ }^{2}$ Director, Vaagdevi College of Engineering, Warangal, A.P., INDIA \\ prof_kkrarediffmail.com
}

\begin{abstract}
This paper describes a frame work investigating the performance of distributed spatial multiplexing (DSM) in cooperative multi-hop cellular networks. The cooperative communication in cellular networks gives us leverage to get the inherent advantages of its random relay locations and the direction of the data flow. However, traditional centralized relay selection needs considerable overhead and signaling. In our proposed work, threshold based relay selection is adopted based on the received signal strength (RSS) and Signal to Noise Ratio (SNR). The best relay chosen will transmit jointly with source using Amplify and forward $(A F)$ protocol. The evaluation is performed with bit error rate (BER) and energy per bit for distributed spatial multiplexing scheme with multi-hop networking.
\end{abstract}

\section{KEYWORDS}

Amplify and Forward relaying, Received signal strength, Energy per bit, Distributed Spatial Multiplexing, Cooperative multi-hop networking, Cellular networks.

\section{INTRODUCTION}

Cooperative communication has recently emerged as a promising technique to combat fading in wireless networks. It leverages the broadcast nature of wireless channel and enables multiple wireless terminals to assist each other for high quality transmission [1]. Although the advantages of Multiple-input-multiple-output (MIMO) systems are well known, it may be impractical to equip very small mobile equipments with multiple antennas. This is primarily due to the size and power limitations of these nodes. To overcome these issues and embrace the benefits offered by the MIMO systems, the concept of cooperative relaying can be successfully implemented in cellular communications [2].

Several cooperation strategies with different relaying techniques, including amplify-and-forward (AF), decode-and-forward (DF), and selective relaying (SR), have been proposed and investigated in terms of outage probability and bit error probability [4]. Next generation multihop cellular networks, especially in the infrastructure based relay networks, multiple concurrent transmission from users are expected and should be well supported by relay stations. The selection of subset relays according to the performance metric can further enhance the DOI : $10.5121 /$ ijcsea.2011.1607 
performance of the cooperative networks [3]. However, how to select the best relays for cooperative diversity in a dynamic network environment is still an open challenge. In particular the work in [5] evaluates the cooperative diversity performance when the best relays are chosen according to the average SNR, and the outage probability. Some researchers have proposed a centralized solution where a fixed node decides which relay will be selected to help the source to forward its information based on perfect knowledge of channel information. In [6], centralized power allocation schemes are presented by assuming all the relay nodes to minimize the system outage behavior and improve the average throughput. In [7], the problem of power allocation has been considered for multiple relay systems. The power allocation schemes in [8] divide the transmit power among the source and the relay(s) to maximize the channel capacity or the instantaneous SNR and thus minimizing the system outage probability. Those schemes have high computation and feedback requirements and cannot be applied for cellular system. However, most existing work focuses on relay selection in a centralized fashion. There is already a lot of works related to cluster formation [4],[7], capacity improvement [9] and energy efficient cooperative communication [3],[10], but little attention has been given to cooperative relay selection on the basis of their location in clusters to improve the energy efficiency and reliability.

In this paper, we propose a completely distributed relay selection scheme for multi-hop cellular networks using Amplify and Forward (AF) protocol based only on the source-relay channel and threshold based optimization. Further this paper describes the idea of distributed spatial multiplexing in wireless multi-hop networks.

The remainder of this paper is organized as follows: In Section II, we describe the system model with threshold based relay selection algorithm for multi-hop cellular networks. The implementation of distributed spatial multiplexing and the evaluation of energy consumption are described in Section III. In Section IV, numerical results are presented and in Section V, we conclude.

\section{SYSTEM MODEL}

Fig. 1, represents a cooperative multi-hop cellular network with Amplify and Forward relaying protocol. The system model considered in this paper is High Speed Uplink Packet access (HSUPA), Release-6. All nodes are assumed to be equipped with single antennas, operating in half duplex mode and each transmission link between any two nodes are modeled as Ricean fading channel. In the general cooperative phenomenon, source broadcasts the information to selected relays and Base station [BS] in phase 1and from phase 2 relays retransmit the amplified version of information to next selected best relays in neighboring cluster. This process continues in multiple phases until relays retransmit the information to BS with minimum acceptable bit error rate $[\mathrm{BER}]$.

\subsection{Threshold based relay selection}

Energy efficiency of cooperative communication in a clustered network has been investigated in [11]. In [11], nodes collaborate on signal transmission and/ or reception in a deterministic way. It is shown that if the long haul transmission distance between clusters is large enough, cooperative transmissions can dramatically reduce the total energy consumption. Energy allocation can further improve the performance of cooperative communication. Optimal energy distribution among cooperative nodes is studied in [12] to minimize the link outage probability. In amplify and forward cooperation protocol, considerable energy saving can be obtained even if source contain few channel information bits [6]. 
Cooperative communications in wireless networks can be more beneficial if transmit cooperative relays are at equal distance from the intended receiver. Propagation measurements in a mobile radio channel indicate that the average received signal strength at any point decays as a power law of the distance of separation between a transmitter and receiver.

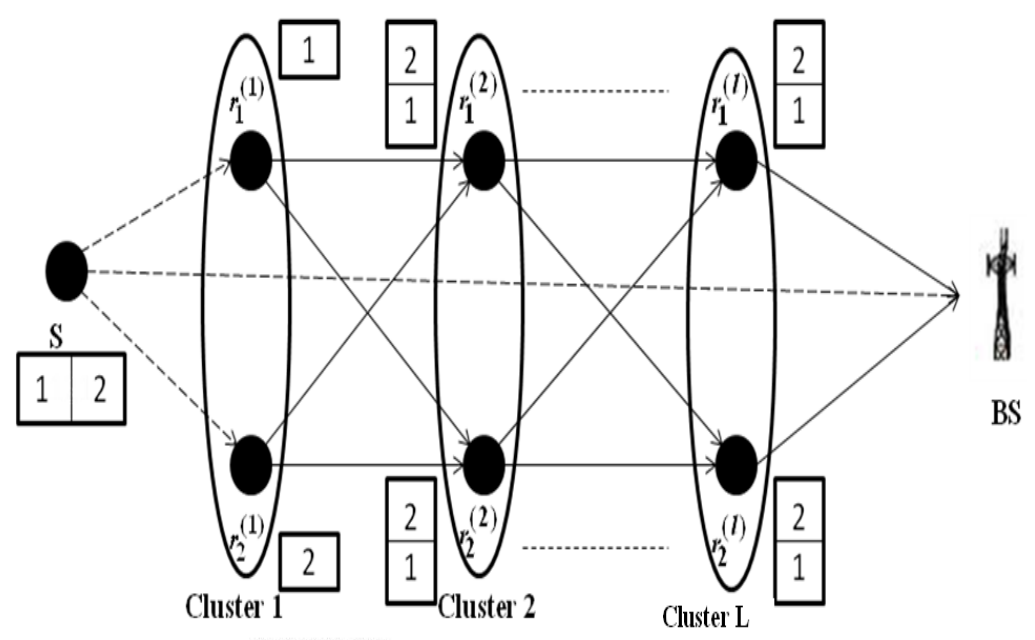

Figure 1. Cooperative Multi-hop Distributed spatial multiplexing cellular network

The average received signal strength (RSS) at a distance $d$ from the transmitting antenna is approximated by

$$
R S S=P_{0}\left(\frac{d}{D_{i}}\right)^{-n}
$$

where $P_{0}$ is the power received at a close in reference point, $n$ is the path loss exponent. $D_{i}$ is the distance of the $i^{\text {th }}$ interferer from the mobile, the received power at a mobile due to the $i^{\text {th }}$ interfering cell will be proportional to $\left(D_{i}\right)^{-n}$.

Based on these distance estimates source allocates an equal-power group identity (Cluster) to those nodes which lies within a certain range, $\mathrm{K}=\min \left(\mathrm{d}_{\mathrm{X} \_\mathrm{S}}(\mathrm{i})\right) \subseteq \mathrm{X}(\mathrm{i})$, where $\mathrm{K}$ represents number of nodes in each cluster. In addition to this the cooperative nodes should be at minimum distance from each other to save energy in local communication within the cooperative relays cluster. We propose Threshold based relay selection algorithm, which generalizes threshold digital relaying to multiple relays. The relay who's SNR larger than Threshold (T) are allowed to retransmit.

The PDF of the SNR is given as

$$
f_{S N R}, l(x)=\frac{1}{\lambda_{k, l}} \exp \left(-\frac{x}{\lambda_{k, l}}\right)
$$

where $S N R_{k, l}$ is the signal to noise ratio on the link between two nodes $k$ and $l, \lambda_{k, l}=E\left(S N R_{k, l}\right)$ and $E($.$) is the expectation operator. Assume that A$ is the number of relays having an SNR greater than the threshold $T$. The probability that $A$ equal to two, means that only two relays are participating in cooperative transmission. 
International Journal of Computer Science, Engineering and Applications (IJCSEA) Vol.1, No.6, December 2011

$$
P\{A=2\}=\sum_{i=1}^{N} P\left(S N R_{S, R_{i}} \geq T\right) \prod_{K=1, K \neq i}^{N} P\left(S N R_{S, R_{K}}<T\right)
$$

\section{EVALUATION OF ENERGY CONSUPTION}

In order to achieve high throughput and spectral efficiency we split the data stream into several sub-streams and transmit the sub-streams simultaneously over spatial sub-channels. These subchannels are created in a distributed fashion by the network itself.

\subsection{Distributed Spatial Multiplexing}

Consider a wireless network as shown in fig. 1, where a single-antenna source wants to send a data packet of length $T_{P}$ to a single-antenna destination over $L+1$ hops, whereas $L$ denotes the number of clusters containing $K$ intermediate nodes that act as single-antenna amplify-andforward (AF) relays. The nodes $r_{1}^{(1)}$ and $r_{2}^{(1)}$ cooperate such, that $r_{1}^{(1)}$ forwards the first half of the data packet and $r_{2}^{(1)}$ the second half. As a result the packet duration at intermediate hops is $T_{P} / 2$. Intermediate nodes simply store and forward the received packets simultaneously without further cooperation. Note that the sub-packets forwarded by $r_{1}^{(2)}$ and $r_{2}^{(2)}$ are linear superposition of the sub-packets forwarded by $r_{1}^{(1)}$ and $r_{2}^{(1)}$. In the last hop the nodes $r_{1}^{(4)}$ and $r_{2}^{(4)}$ cooperate such, that the destination receives two sub-packets sequentially in time. With suitable space-time signal processing at the source, the destination is able to decode the information bits. As the packet length reduces to $T_{P} / 2$, the multi-hop delay drops accordingly. Neglecting the time for signal propagation, the multi-hop delay $\tau$ is in general given by $\tau=T_{P}\left(2+\frac{L-1}{K}\right)$, where $K$ is the number of forwarding relays in a cluster.

\subsubsection{Signal and Channel Model}

All signal arrivals and departures are perfectly synchronized. The nodes cannot transmit and receive simultaneously and transmitted signals only reach the next cluster. The equivalent complex baseband signals received by the relays in the first cluster are stacked into a vector $y_{1}=H_{1} x+w_{1}$ where $x=\left(x_{1}, \ldots, x_{K}\right)^{T}$ is the data vector sent by the source with average power constraint $E\left[\left.\left|x_{i}^{2}\right|\right|_{=} / K\right.$. Note that $x_{i}$ is the symbol which will be forwarded from relay $i$ in the first cluster. $\mathrm{H}_{1}=\operatorname{diag}\left(h_{11}, \ldots . ., h_{K 1}\right)$ is a $\mathrm{KxK}$ diagonal matrix with $h_{i 1} \approx C N(0,1)$ the complex path gain from source to relay $i$ in the first forwarding cluster and $\mathrm{w}_{1}=\left(w_{1}^{(1)}, \ldots, w_{K}^{(1)}\right)^{\mathrm{T}}$ with $w_{i}^{(l)} \approx C N\left(0, \sigma_{R}^{2}\right)$ describes the additive white Gaussian noise at the relays. The symbols $x_{1}, \ldots . ., x_{K}$ are launched into the network via orthogonal channels that are described by $\mathrm{H}_{1}$. The signals received in the $l$ th cluster are given as $\mathrm{y}_{l}=\mathrm{H}_{l} \mathrm{~g}_{l} \mathrm{y}_{l-1}+\mathrm{w}_{l}$, with $\mathrm{y}_{l}=\left(y_{1}^{(l)}, \ldots . ., y_{K}^{l}\right)^{\mathrm{T}} . \mathrm{H}_{l}$, 
where $l=2, \ldots \ldots . . ., L$ is a $K \times K$ channel matrix with elements $h_{i j}^{(l)} \approx C N(0,1)$ describing the complex path gain from the $j$ th relay in cluster $l-1$ to the $i$ th relay in cluster $l$. The scaling factor $g_{l}$ is chosen such that the average transmits power of every relay equals $P / K$. The vector received by the destination is then given by

$$
\mathrm{y}=\underbrace{\mathrm{H}_{L+1} \prod_{l=1}^{L} \mathrm{~g}_{l} \mathrm{H}_{l} \mathrm{x}}_{\mathrm{H}} \underbrace{\sum_{m=1}^{L} \prod_{n=m}^{L} \mathrm{~g}_{n} \mathrm{H}_{n+1} \mathrm{w}_{m}+\mathrm{w}}_{\mathrm{n}}
$$

With

$$
\mathrm{g}_{l}^{2}= \begin{cases}\frac{P / K}{P / K+\sigma} R^{2} & ; l=1, \\ \frac{P / K}{P+\sigma_{R^{2}}} & ; 2 \leq l \leq L .\end{cases}
$$

$\mathrm{H}_{\mathrm{L}+1}=\operatorname{diag}\left(h_{11}, \ldots . ., h_{1 K}\right)$ is again a $K \times K$ diagonal matrix with $h_{1 i} \approx C N(0,1)$ the complex path gain from relay $i$ in the last forwarding cluster to the destination. The covariance matrix $\mathrm{R}$ is given as

$$
\mathrm{R}=\sum_{m=1}^{L} \prod_{n=m}^{L} \mathrm{~g}_{n}^{2} \mathrm{H}_{n+1}\left(\prod_{n=m}^{L} \mathrm{H}_{n+1}\right)^{\mathrm{H}} \sigma_{\mathrm{R}}^{2} \mathrm{I}_{K}+\sigma^{2} \mathrm{I}_{K}
$$

Where $\sigma^{2}$ denotes the noise power at the destination.

\subsection{Evaluation for Energy per Bit}

We have modeled the cellular network with Ricean fading channel model. In the particular arrangement of cooperative multi-hop network shown in Fig. 1, where all relays are at same distance from the intended receivers, following equation (6) represents the total energy required per bit per hop,

$$
\begin{aligned}
E_{L+1 \_b i t}(i)= & E_{L_{-} b i t}(i)+(1+\alpha) \frac{\left(4 \pi d_{X} S^{(i)}\right)^{2}}{G_{t} G_{r} \lambda^{2}} M_{l} N_{f} \\
& +\frac{P_{t x_{-} \text {elect }}}{R_{b}} n_{T}+\frac{P_{r x_{-} \text {elect }}}{R_{b}} n_{R}
\end{aligned}
$$

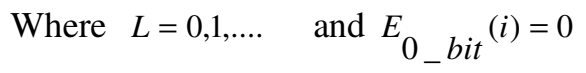

where $\alpha$ is depends upon the drain efficiency of power amplifier in transmitter circuitry, $\rho$ is the signal power to noise ratio at each of the receive node, $N_{0}$ is AWGN power spectral density, $d_{X_{-} S}(i)$ is the average distance between transmitter and receiver, $\lambda$ is the carrier wavelength, $G_{t}$ $G_{r}$ are antenna gains of transmitter and receiver respectively, $M_{l}$ is the link margin compensating hardware process variations and other noise or interference, $N_{f}$ is the receiver noise figure, $R_{b}$ is transmission rate and $P_{t x_{-} \text {elect }}(180 \mathrm{~mW}), P_{r x_{-} \text {elect }}(200 \mathrm{~mW})$ are power dissipated in transmitter and receiver circuits, respectively. The transmitted power depends on $\rho$ and bit error 
International Journal of Computer Science, Engineering and Applications (IJCSEA) Vol.1, No.6, December 2011 probability, $P_{b}(\rho) \approx \operatorname{erfc}(\sqrt{a \rho}), \quad$ where $\quad \mathrm{a}, \mathrm{b}>0 \quad$ and $\quad$ SNRs $\quad$ of the $S-B S, S-K(i)$ and $K(i)-B S$ links are denoted by $\rho_{S-B S}, \rho_{S-K(i)}, \rho_{K(i)-B S}$ respectively.

Note that typically $b$ depends on the minimum distance in the consolation and depends on the number of neighbors with minimum distance. The bit error probability of most popular schemes can approximated as, for BPSK $(\mathrm{b}, \mathrm{a})=(0.5,1)$ and for MPSK $(b, a)=\left(1 / \log _{2}(M), \log _{2}(M) \sin ^{2}(\pi / M)\right)$. Based on this general $P_{b}$ expression, the average bit error probability under Ricean fading is expressed as

$$
\begin{aligned}
\overline{P_{b}}(\rho)= & E_{\rho}[\operatorname{erfc}(\sqrt{a \rho})]=b\left[1-\sqrt{\frac{a \rho}{1+a \rho}}\right] \\
E_{L+1 \_b i t}(i)= & E_{L \_b i t}(i)+(1+\alpha) \frac{n_{T} \ln \left|1 / \bar{P}_{b}\right|_{0} N_{0}}{4 \mid H_{L+1} \|_{F}^{2}} \frac{\left(4 \pi d_{X \_} S^{(i)}\right)^{2}}{G_{t} G_{r} \lambda^{2}} M_{l} N_{f} \\
& +\frac{P_{t x \_ \text {elect }}}{R_{b}} n_{T}+\frac{P_{r x \_l e c t}}{R_{b}} n_{R}
\end{aligned}
$$

Therefore, the bit error rate probability and energy per bit will eventually converge to a value solely determined by the distance between the neighboring relays, modulation schemes and the respective SNRs.

\section{NUMERICAL RESULTS}

To demonstrate the efficiency of the proposed framework, we assume the proposed network is High Speed Up-link Packet Access (HSUPA), Release-6 (category 6). BW=2000MHz, Transmitted power of UE $=21 \mathrm{dBm}$ (Class $4 \mathrm{UE}$ ), number of multi-hops (Phases) $=3$. Data rate $=5.8 \mathrm{Mbps}$, Radius of cell site $=600 \mathrm{~m}$, and the number of data bits in a packet $=1024 \mathrm{bits}$.

Fig. 2 depicts the computational experiments carried out on a set of moderately sized network with 50 random nodes, distributed in 600X600 grid.

Xloc= 8.95114 .25233 .45232 .15436 .5515 .26514 .23218 .2319 .0216 .223 .2524 .6296 .5 328.5429 .4217 .56325 .2638 .45336 .24314 .21312 .45452 .2419 .2471 .5462 .2412 .3562 .2 582.6512 .2593 .26646264563743753725788248684939698959177988433 .3120 111136145156166171183194164200210221234241253264275283294299.12301 310320330349358362372383387399402419423438447455465476487498503512 523533544566577588599600 .

Yloc $=599.5165 .32287 .25129 .63545 .25569 .25415 .25515 .63134126222123 .6120 .5150$ 31633542522642830634045460484225115335186465626971375273179848586 919596981216194753.92382590560555456432345456234345456567243354576 262363474575233244255266277245455466477488499453452451354356357358 328421487365376385393330350337358390590570560520.

Fig. 3, represents threshold based selected nodes in each cluster. The number of clusters are confined to $\mathrm{L}=3$ for simplicity. The cluster and best two relays are selected based on distance and threshold criteria. Fig. 4, provides an analysis of Distributed Spatial Multiplexing (DSM) bit error rate at selected multi phases. Because of the Amplify and Forward relay protocol implementation 
International Journal of Computer Science, Engineering and Applications (IJCSEA) Vol.1, No.6, December 2011

in DSM, it is observed from various iterations that BER increases with number of multi phases. Fig 5 and 6, demonstrates the energy consumption per bit for multiple phases, selected at different locations.

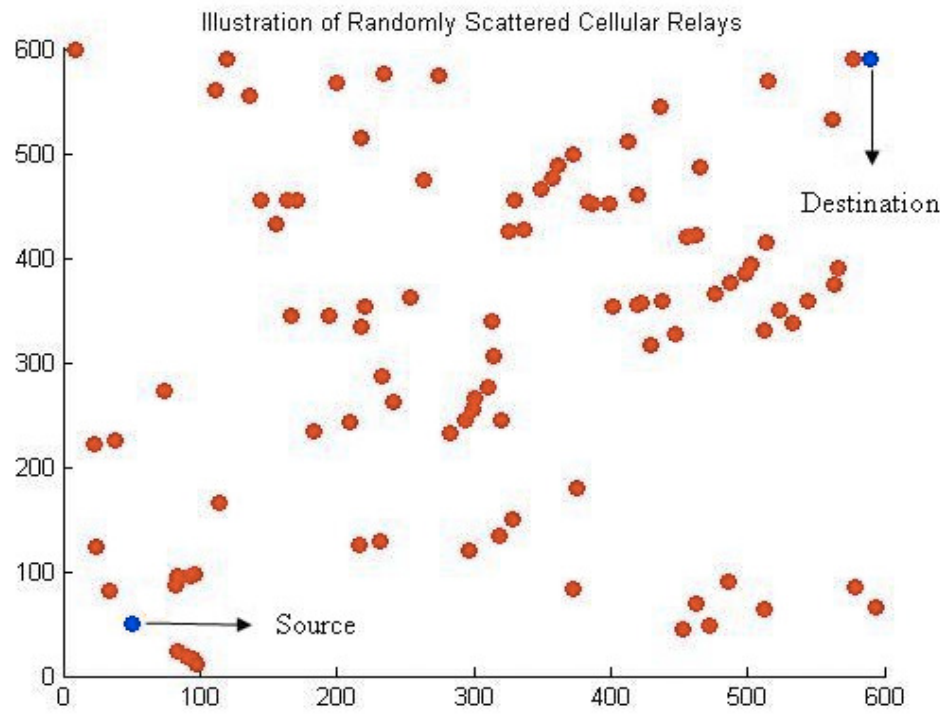

Figure 2. Illustrating randomly spaced cellular nodes

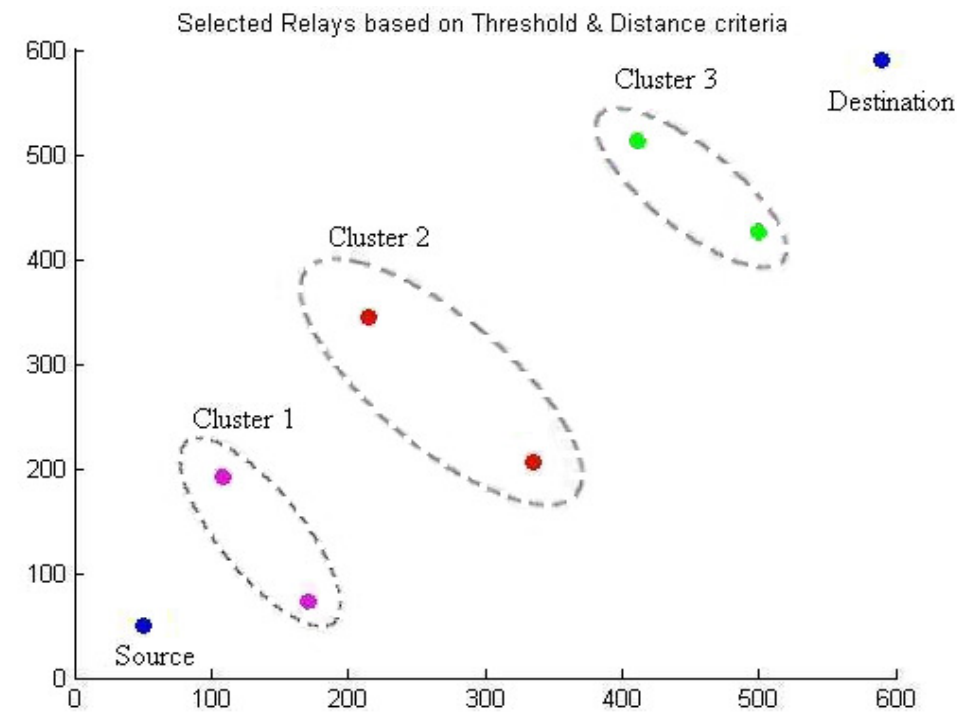

Figure 3. Selected Clusters and best relays based on proposed Algorithm 
International Journal of Computer Science, Engineering and Applications (IJCSEA) Vol.1, No.6, December 2011

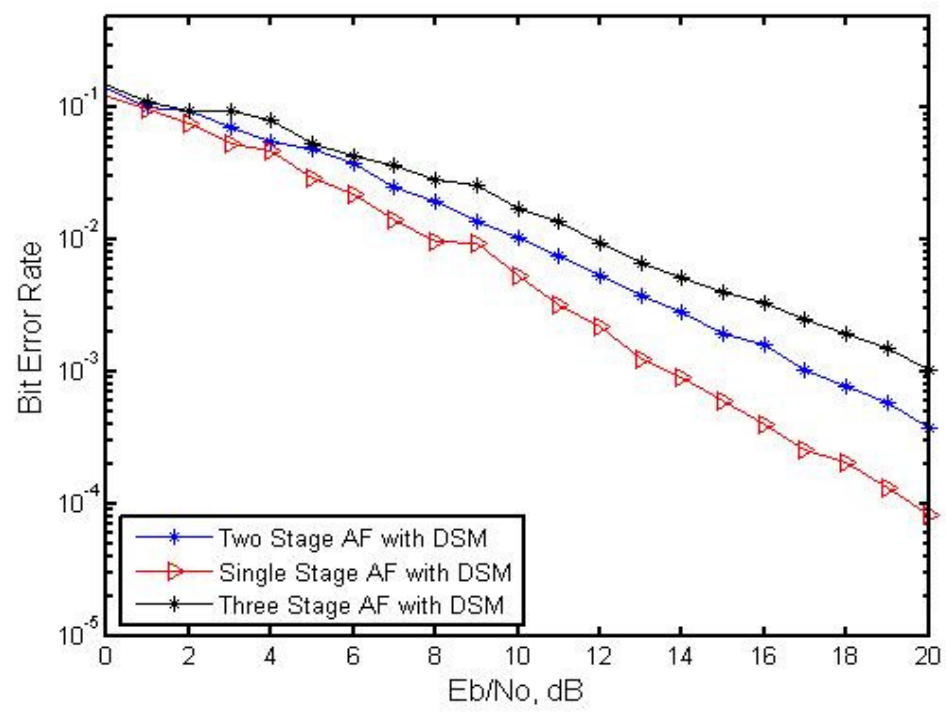

Figure 4. Performance of Distributed Spatial Multiplexing in multi-hop AF relaying protocol

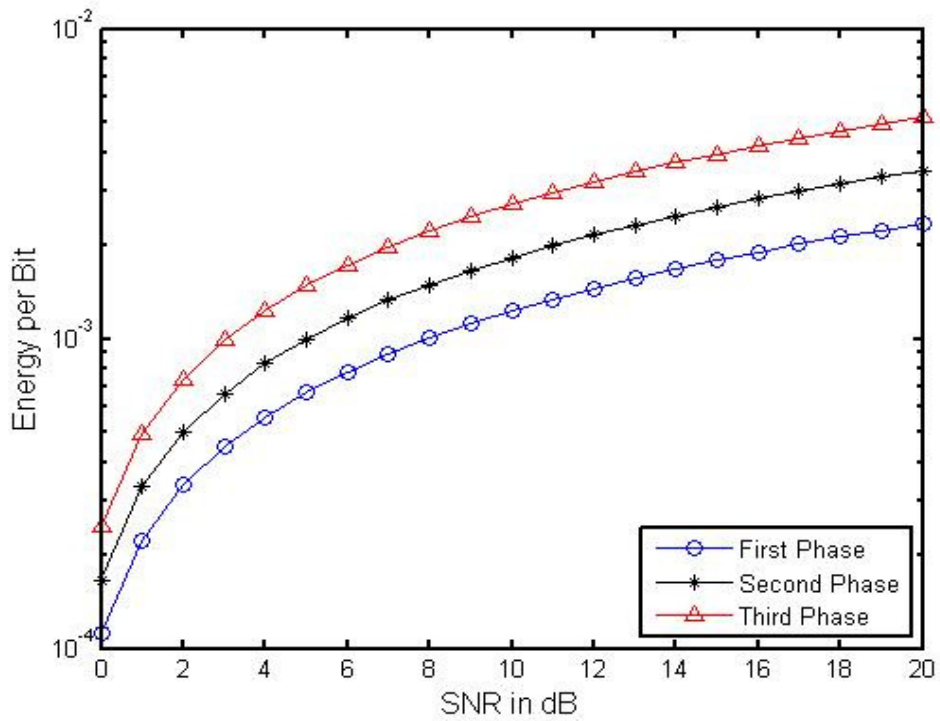

Figure 5. Evaluation of Energy per bit versus SNR 


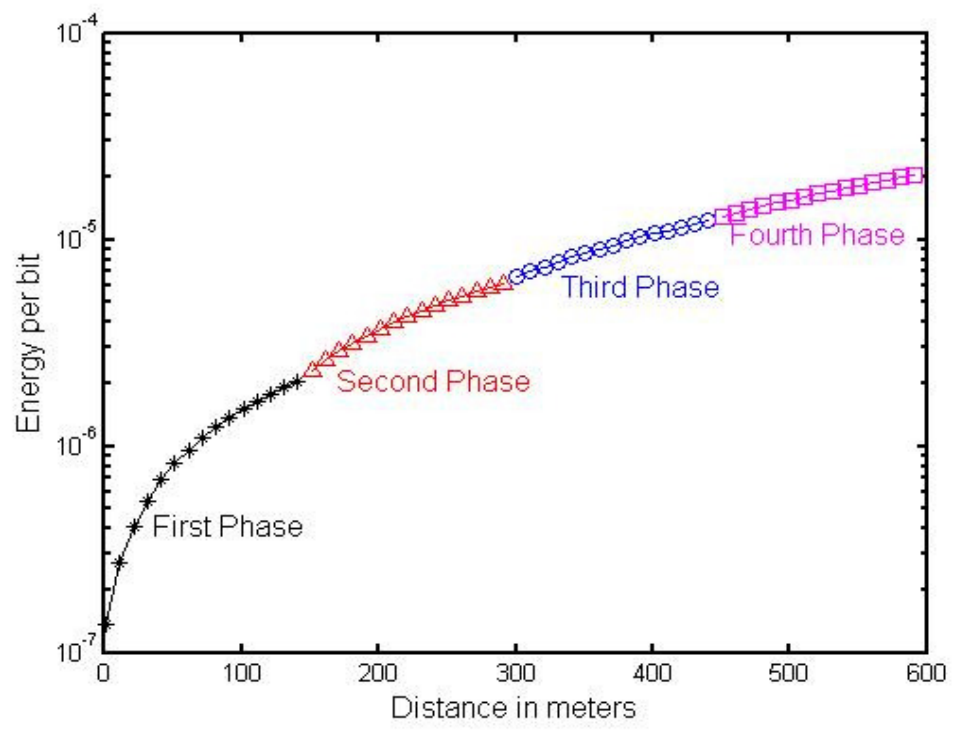

Figure 6. Evaluation of Energy per bit with multi-hop distance metric

\section{CONCLUSION}

In this paper, we provided a frame work on multi-hop cooperative cellular network, in which the evaluation of the system is carried out in two phases. Threshold based relay selection Algorithm is proposed with less complexity and fairness for cellular networks. Furthermore, Distributed Spatial Multiplexing (DSM) with Amplify and Forward relaying is analyzed for multi-hop networks. The numerical results demonstrated the energy consumption per bit for both schemes under distance and diversity metrics.

\section{REFERENCES}

[1] A.Sendonaris, E.Erkip and B.Aazhang,"User Cooperation Diversity Part I and Part II," IEEE Trans. Commun., vol. 51,no.11, Nov.2003,pp.1927-48.

[2] M.Sushanth Babu and Prof. K.Kishan Rao, "Performance Analysis of Cooperative wireless Networks with distributed coding", International Journal of Advanced Communication Engineering, Vol.3, no.1, June 2011, pp.103-108.

[3] T.W.Ban, B.C.Junge, D.K.Sung, and W.Choi, "Performance Analysis of Two Relay Selection Schemes for Cooperative Diversity", in Proc. IEEE PIMRC. Athens, Greece, Sept.2007.

[4] J.Laneman, D.Tse, and G. Wornell, "Cooperative Diversity in Wireless Networks: Efficient Protocols and Outage Behaviour," IEEE Trans. Commun. Inf. Theory, vol. 50, no. 12, pp. 3062-3080, Dec. 2004.

[5] J. Luo, R. S. Blum, L.J. Greenstein, L.J. Cimini and A.M. Haimovich, "New Approaches for Cooperative use of Multiple Antennas in Ad-hoc Wireless Networks", in Proc, IEEE Vehicular Technology Conference, vol. 4, pp. 2769-2773, Sept. 2004.

[6] Y. Zhao, R. Adve, and T.J.Lim, "Improving Amplify-and-Forward Relay Networks: Optimal Power Allocation versus Selection," IEEE International Symposium on Inf. Theory, pp.1234-1238, July 2006.

[7] Y.Chen, G.Yu, P. Qiu, and Z. Zhang, "Power-aware Cooperative relay Selection strategies in wireless ad-hoc networks," in Proc. IEEE PIMRC, Helsinki, Finland, Sept. 2006. 
International Journal of Computer Science, Engineering and Applications (IJCSEA) Vol.1, No.6, December 2011

[8] H.C.Yang and M.S. Alouini," A power saving implementation of generalized selection combining by output thresholding," in Global Telecommunications Conference, GLOBECOM'04. IEEE, vol. 6, pp. 3979-3983, Dec. 2004.

[9] K.T. Phan, T.Le-Ngoc, S.A.Vorobyov, and C. Tellambura,"Power allocation in wireless multi-user relay networks." IEEE Transactions on Wireless Communications, vol.8, pp. 2535-2545. Nov. 2009.

[10] A.Bletsas, H.Shin, and M.Z.Win, "Cooperative communications with outage-optimal opportunistic relaying," IEEE Trans. on Wireless Communications, vol.6, pp. 3450-3460, June 2007.

[11] Wenfeng Li; Weike Chen; Xinzhu Ming, "A Local-centralized Adaptive Clustering Algorithm for Wireless Sensor Networks," Proc. -15th Int'l Conf. on Computer Comm. and Networks, Oct. 2006 Page(s): 149 - 154.

[12] L.Simic, S.M.Berber and K.W.Sowerby, "Partner choice and power allocation for energy efficient cooperation in wireless sensor networks," in Proc. IEEE ICC2008, pp. 4255-4260, May 2008.

\section{Authors}

M.SUSHANTH BABU received his B.E. in Electronics and Communication Engineering in 2002 from North Maharastra University and his M.Tech. degree from Jawaharlal Nehru Technological University, Hyderabad in 2008. He has been working towards his Ph.D. degree in Wireless Communications at Jawaharlal Nehru Technological University, Hyderabad since 2009. He is presently working as Associate Professor in Department of Electronics and Communication Engineering. He guided 18 Masters and 30 UG projects. He is a member of professional bodies like, IEEE, ISTE and IETE. He is technical program committee member of 09 IEEE International Conferences. His research interests are in the areas of Wireless Mobile Communication, Cellular Networking, Distributed Cooperative Communication, MIMO and Signal Processing Applications.

Prof.K.KISHAN RAO is currently a Professor in Electronics and Communication Engineering and working as Director in Viswambhara Educational Society. He received his B.E. and M.E. degrees from Osmania University in 1965 and 1967.He is awarded with Ph.D. degree from Indian Institute of Technology, Kanpur [IIT] in 1973.He worked as Principal for National Institute of Technology and Kakathiya Institute of Technology and Science, Warangal. He a senior member of professional bodies like IEEE [comsoc], ISTE, and IETE. He has published over 78 International articles. He currently serves as

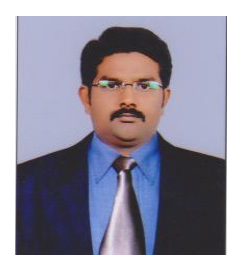
Editor for International Journal of Wireless Personal communication, Springer and International Journal of Wireless Networks, Springer. He guided 03 Ph.D scholars and guided 62 Master Projects. His research interests are in the areas of Wireless Communications, Signal Processing Applications and Cooperative Mobile Communications. 